THE DIFFERENCE BETWEEN GENERATION Y AND GENERATION Z ABOUT MOTIVATIONS OF SHARING TRAVEL PHOTOS ON FACEBOOK

Le Thai Phuong

Da Nang Architecture University

\begin{tabular}{|c|c|c|}
\hline \multicolumn{2}{|c|}{ ARTICLE INFO } & ABSTRACT \\
\hline Received: & 21/1 & Taking photos and sharing travel photos on Facebook is gaining \\
\hline Revised: & 18/11/2021 & $\begin{array}{l}\text { popularity among travelers. The study aims to identify and analyze the } \\
\text { differences between Generation } \mathrm{Y} \text { and Generation } \mathrm{Z} \text { about motivations }\end{array}$ \\
\hline Published: & 18/11/2021 & of sharing travel photos on Facebook. Cronbach's Alpha, Exploratory \\
\hline & & Factor Analysis and Confirmatory Factor Analysis were used to \\
\hline \multicolumn{2}{|l|}{ KEYWORDS } & identify the motivations. Independent $T$ - Test is used to assess the \\
\hline \multicolumn{2}{|l|}{ Motivation } & obtained from a survey of 584 travelers showed that the 5 motivations \\
\hline \multicolumn{2}{|l|}{ Sharing travel photos } & for sharing travel photos on Facebook of Generation $\mathrm{Y}$ and $\mathrm{Z}$ are: Self- \\
\hline \multicolumn{2}{|l|}{ Facebook } & expression, information, entertainment, disclosure, image retention. In \\
\hline \multicolumn{2}{|l|}{ Generation Y } & $\begin{array}{l}\text { ntion are two prominent motivations } \\
\text { and entertainment are two prominent }\end{array}$ \\
\hline \multicolumn{2}{|l|}{ Generation $\mathrm{Z}$} & tivations of Generation $\mathrm{Z}$. \\
\hline
\end{tabular}

\title{
SỰ KHÁC BIỆT GIŨ̉A THẾ HỆ Y VÀ THẾ HỆ Z VỀ ĐộNG CƠ CHIA SẺ HİNH ẢNH DU LỊCH LÊN FACEBOOK
}

Lê Thái Phượng

Truòng Đại học Kiến trúc Đà Nã̃ng

\begin{tabular}{|c|c|}
\hline THÔNC & \\
\hline Ngày nhận bài: $21 / 10 / 2021$ & \multirow{9}{*}{ 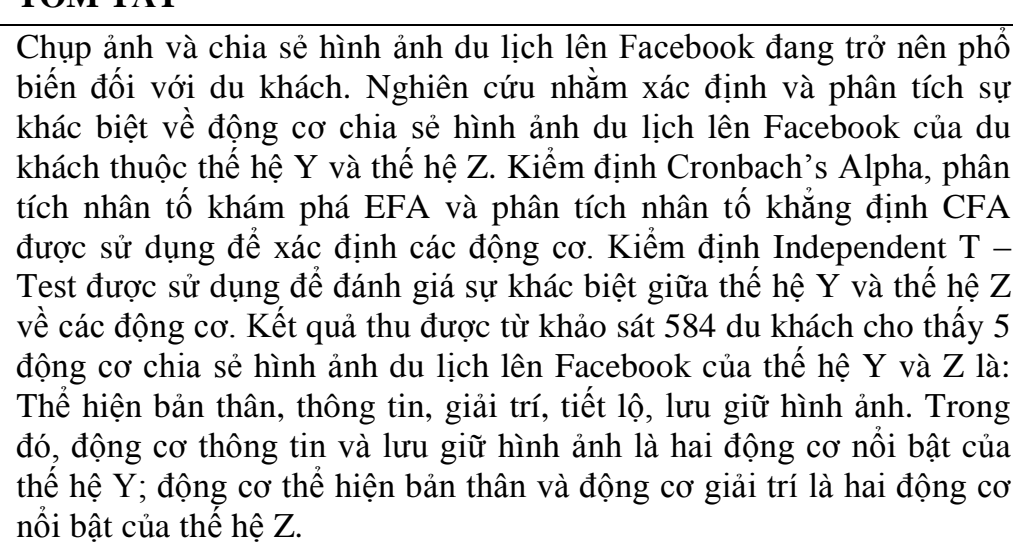 } \\
\hline Ngày hoàn thiện: $18 / 11 / 2021$ & \\
\hline Ngày đăng: $18 / 11 / 2021$ & \\
\hline TÙ KHÓA & \\
\hline Động cơ & \\
\hline hia sẻ hình ả & \\
\hline acebook & \\
\hline e nẹ Y & \\
\hline Thế hệ Z & \\
\hline
\end{tabular}

DOI: https://doi.org/10.34238/tnu-jst.5194

Email: phuonglt@dau.edu.vn 


\section{1. Đặt vấn đề}

Chụp ảnh khi đi du lịch đã xuất hiện từ lâu bởi du lịch luôn gắn liền với những địa điểm nổi tiếng, những phong cảnh đẹp hay những nét văn hóa, phong tục độc đáo... Du khách thường chụp ảnh để ghi lại kinh nghiệm du lịch cá nhân và chia sẻ kinh nghiệm du lịch cá nhân của họ với những người khác [1]. Ngày nay, sự phát triển của kinh tế và khoa học công nghệ khiến cho việc chụp ảnh khi tham quan du lịch càng trở nên thuận lợi và phổ biến hơn. Sự sẵn có rộng rãi của Internet và các thiết bị di động với camera tích hợp đã cho phép du khách chia sẻ trải nghiệm của họ với người khác bằng cách đăng ảnh lên mạng xã hội [2]. Chia sẻ hình ảnh đang trở thành một trong những hoạt động phổ biến nhất trên mạng xã hội [3]. Theo Advertising Vietnam (2021), mỗi ngày có khoảng 350 triệu bức ảnh được đăng tải lên mạng xã hội bao gồm cả tài khoản cá nhân và các trang doanh nghiệp [4]. Từ việc đáp ứng nhu cầu tự thân của khách du lịch, việc chụp ảnh khi đi du lịch và chia sẻ hình ảnh lên mạng xã hội vô tình đã trở thành công cụ quảng bá rất hữu hiệu cho du lịch.

Theo Omnicore (2021), tính đến tháng 6 năm 2021, tổng số người dùng hoạt động hằng ngày trên Facebook là 1,88 tỷ; người dùng ở độ tuổi từ 25 đến 35 chiếm tỷ lệ nhiều nhất $(31,6 \%)$, sau đó là độ tuổi từ 18 đến $24(23,8 \%)$ [5]. Hiện nay, Việt Nam là quốc gia đứng thứ 7 trên thế giới về số lượng người dùng Facebook, tính đến tháng 6 năm 2021, tổng số người dùng Facebook tại Việt Nam là gần 76 triệu người, chiếm hơn $70 \%$ dân số, tăng 31 triệu người dùng so với năm 2019 và vẫn dẫn đầu danh sách các mạng xã hội phổ biến tại Việt Nam [6].

Chính sự phát triển của nhu cầu chụp ảnh du lịch và sự phát triển của mạng xã hội đã khiến hoạt động chụp ảnh du lịch được các nhà nghiên cứu quan tâm. Trên thế giới đã có nhiều công trình nghiên cứu ở các khía cạnh khác nhau, chẳng hạn như: Hành vi chụp ảnh trong du lịch [7][10]; vai trò của chụp ảnh trong du lịch [11]-[14]; hành vi chụp ảnh du lịch và chia sẻ trực tuyến [1], [2], [15], [16]. Một số nghiên cứu cũng đề cập đến sự khác biệt về đặc điểm nhân khẩu học trong hành vi chia sẻ hình ảnh du lịch lên mạng xã hội [17], [18], [3], [2]. Mặc dù hành vi chụp ảnh và chia sẻ hình ảnh du lịch đã được nghiên cứu trước đây nhưng những nghiên cứu về động cơ chia sẻ hình ảnh du lịch lên mạng xã hội còn hạn chế [2] và chưa có nghiên cứu nào làm rõ động cơ chia sẻ hình ảnh du lịch lên mạng xã hội ở các thế hệ khác nhau. Tại Việt Nam, chưa có bất kỳ nghiên cứu nào về chia sẻ hình ảnh du lịch lên mạng xã hội nên đây là một khoảng trống nghiên cứu lớn. Nghiên cứu này tập trung vào Facebook, là mạng xã hội phổ biển nhất tại Việt Nam và du khách là những người thuộc thế hệ $Y$ và $Z$. Những người thuộc thế hệ $Y$ có năm sinh từ 1980 đến 1995 (26 tuổi đển 41 tuổi), những người thuộc thể hệ $Z$ có năm sinh từ 1996 đến 2010 (11 tuổi đến 25 tuổi) [19]. Qua đó, nghiên cứu làm rõ sự khác biệt trong động cơ chia sẻ hình ảnh du lịch lên Facebook ở hai thế hệ. Việc hiểu được động cơ chia sẻ ảnh của du khách có ý nghĩa rất quan trọng đối với ngành du lịch, bao gồm quản lý hình ảnh điểm đến, phát triển chiển lược tiếp thị và cải thiện sự hài lòng của du khách [1].

\section{Phương pháp nghiên cứu}

\subsection{Thang đo động co chia sẻ hình ảnh du lịch lên Facebook}

Động cơ được hiểu là điều thúc đẩy con người hoạt động nhằm thỏa mãn nhu cầu, là động lực kích thích trực tiếp, là nguyên nhân của hành vi [20]. Như vậy, động cơ chia sẻ hình ảnh du lịch lên Facebook có thể được hiểu là nguyên nhân thúc đẩy, kích thích du khách chia sẻ hình ảnh du lịch lên Facebook.

Chia sẻ hình ảnh du lịch lên mạng xã hội được các nghiên cứu trước đề cập với nhiều động cơ khác nhau như: Tìm kiếm tình cảm (affection seeking) [17], [21]; tìm kiếm sự chú ý (attention seeking) [22], [23]; tiết lộ (disclosure) [24], [25], [2]; giải trí (entertainment) [22], [26], [2]; thú tiêu khiển theo thói quen (habitual pastime) [26]; chia sẻ thông tin (information sharing) [22], [26], [2]; ảnh hưởng xã hội (social influence) [25]; tương tác xã hội (social interaction) [22], [25], sự công nhận và vị trí xã hội (social recognition \& status) [2]. 
Trong nghiên cứu này, tác giả kế thừa thang đo của Li (2020) gồm 4 động cơ (15 biến quan sát) và bổ sung thêm động cơ lưu giữ hình ảnh với 3 biến quan sát (Bảng 1). Thang đo Likert 5 mức độ được sử dụng: 1 - Hoàn toàn không đồng ý; 2 - Không đồng ý; 3 - Bình thường; 4 Đồng ý; 5 - Rất đồng ý.

Bảng 1. Thang đo động cơ chia sẻ hình ảnh du lịch lên Facebook

\begin{tabular}{|c|c|c|}
\hline Động cơ & Mã hóa & Nguồn \\
\hline 1. Thể hiện bản thân & TH & \multirow{7}{*}[2]{} \\
\hline Tôi chia sẻ ảnh đi du lịch để trở nên nổi tiếng & TH1 & \\
\hline Tôi cảm thấy bản thân được xem trọng khi chia sẻ ảnh đi du lịch & TH2 & \\
\hline Tôi chia sẻ ảnh đi du lịch để được mọi người chú ý & TH3 & \\
\hline Tôi chia sẻ ảnh đi du lịch để được nhiều lượt thích & TH4 & \\
\hline Tôi chia sẻ ảnh đi du lịch để có nhiều bình luận & TH5 & \\
\hline Vị trí xã hội của tôi sẽ tăng lên khi chia sẻ ảnh đi du lịch & TH6 & \\
\hline 2. Thông tin chuyến đi & TT & \multirow{5}{*}[2]{} \\
\hline Tôi chia sẻ ảnh đi du lịch để chia sẻ những thông tin về chuyến đi & TT1 & \\
\hline Tôi chia sẻ ảnh đi du lịch để truyền tải vẻ đẹp của điểm đến & TT2 & \\
\hline Tôi chia sẻ ảnh đi du lịch để chia sẻ những trải nghiệm cá nhân về chuyến đi & TT3 & \\
\hline 3. Giải trí & GTR & \\
\hline Chia sẻ ảnh đi du lịch khiến tôi thấy vui & GT1 & \multirow{3}{*}{ [2] } \\
\hline Tôi cảm thấy hào hứng khi chia sẻ ảnh đi du lịch & GT2 & \\
\hline Chia sẻ ảnh đi du lịch như là một hình thức giải trí & GT3 & \\
\hline 4. Tiết lọ & $\mathbf{T L}$ & \multirow{4}{*}{ [2] } \\
\hline Tôi chia sẻ ảnh đi du lịch để tiết lộ với mọi người về cuộc sống của tôi & TL1 & \\
\hline Tôi chia sẻ ảnh đi du lịch để tiết lộ với mọi người về chuyến du lịch của tôi & TL2 & \\
\hline Tôi chia sẻ ảnh đi du lịch để tiết lộ với mọi người về những người bên cạnh tôi & TL3 & \\
\hline 5. Lưu giữ hình ảnh & GL & \multirow{4}{*}{ Tác giả } \\
\hline Tôi chia sẻ ảnh đi du lịch để sau này xem lại và nhớ về chuyến đi & GL1 & \\
\hline Tôi chia sẻ ảnh đi du lịch để sau này Facebook nhắc lại kỉ niệm về chuyến đi & GL2 & \\
\hline Tôi chia sẻ ảnh đi du lịch để dễ dàng tìm kiếm hình ảnh khi cần & GL3 & \\
\hline
\end{tabular}
(Nguồn: Tổng hơp của tác giả)

\subsection{Mẫu nghiên cúu}

Nghiên cứu sử dụng phương pháp chọn mẫu thuận tiện dựa trên tiêu chí là tính dễ tiếp cận. Bảng câu hỏi có cấu trúc được thiết kế bằng Google Form và thực hiện khảo sát trực tuyến đối với những người có đi du lịch trong giai đoạn 2019 - 2021, có chia sẻ hình ảnh của chuyến du lịch đó lên Facebook.

Thời gian khảo sát chia làm 2 đợt. Đợt 1 từ ngày 02/04/2021 đến 30/06/2021, thu được 430 mẫu đạt yêu cầu, trong đó người thuộc thế hệ $Y$ là 138 người (chiếm 32\%), thế hệ $Z$ là 292 người (chiếm 68\%). Do tỷ trọng người thuộc thế hệ $\mathrm{Y}$ thấp nên cuộc khảo sát đợt 2 chỉ dành cho người thuộc thế hệ Y, thời gian khảo sát từ ngày 01/07/2021 cho đến khi thu được 154 mẫu đạt yêu cầu vào ngày $14 / 08 / 2021$. Như vậy, tổng số mẫu sử dụng trong phân tích là 584 mẫu với tỷ trọng người thuộc thế hệ $\mathrm{Y}$ và $\mathrm{Z}$ bằng nhau. Đặc điểm của mẫu được trình bày ở bảng 2 .

Bảng 2. Thống kê mẫu nghiên cưu

\begin{tabular}{|c|c|c|c|c|c|}
\hline Đặc điểm & Số lượng & Tỷ trọng \% & Đặc điểm & Số lượng & Tỷ trọng \% \\
\hline 1. Giới tính & 584 & 100 & 3. Độ tuổi & 584 & 100 \\
\hline Nam & 236 & 40,4 & Từ 11 đến 18 tuổi & 109 & 18,7 \\
\hline Nữ & 348 & 59,6 & Từ 19 đến 25 tuổi & 183 & 31,3 \\
\hline 2. Nơi ổ & 584 & 100 & Từ 26 đến 30 tuổi & 86 & 14,7 \\
\hline Miền Bắc & 123 & 21,1 & Từ 31 đến 35 tuổi & 124 & 21,2 \\
\hline Miền Trung & 320 & 54,8 & Từ 36 đến 41 tuổi & 82 & 14,0 \\
\hline Miền Nam & 141 & 24,1 & & & \\
\hline
\end{tabular}

(Nguồn: Kết quả xủ lý dũ liệu của tác giả) 


\subsection{Phưong pháp xử lý số liệu}

Phần mềm SPSS 20.0 và $\mathrm{AMOS} 20.0$ được sử dụng để phân tích dữ liệu. Các phương pháp phân tích gồm:

+ Thống kê mô tả

Thống kê mô tả nhằm thống kê đặc điểm mẫu nghiên cứu và làm rõ số lượng các câu trả lời của đối tượng phỏng vấn. Ngoài ra, giá trị trung bình được sử dụng để xác định động cơ chia sẻ hình ảnh du lịch lên Facebook của du khách.

+ Kiểm định giá trị của thang đo

Giá trị của thang đo gồm: Giá trị tin cậy, giá trị hội tụ và giá trị phân biệt. Giá trị của thang đo được kiểm định thông qua phân tích Cronbach's Alpha, EFA và CFA [27].

+ Kiểm định sự khác biệt về trung bình của tổng thể

Sự khác biệt về trung bình của tổng thể được kiểm định bằng phương pháp Independent $\mathrm{T}$ Test [28] nhằm tìm ra sự khác biệt giữa du khách thuộc thế hệ $Y$ và thế hệ $Z$ trong động cơ chia sẻ hình ảnh du lịch lên Facebook.

\section{Kết quả nghiên cứu}

\subsection{Kiểm định Cronbach's Alpha}

Đối với thế hệ Y, biến quan sát TH1 và TH6 có hệ số tương quan biến tổng nhỏ hơn 0,3 nên bị loại, 16 biến quan sát còn lại được tiếp tục phân tích. Kết quả cho thấy thang đo đạt độ tin cậy do hệ số Cronbach's Alpha của các thành phần đều lớn hơn 0,6 và tương quan biến tổng của các biến quan sát đều lớn hơn 0,3 (Bảng 3). Tương tự, đối với thế hệ $\mathrm{Z}$, biến quan sát TH1 có hệ số tương quan biến tổng nhỏ hơn 0,3 nên bị loại, 17 biến quan sát còn lại đảm bảo độ tin cậy. Kết quả phân tích Cronbach's Alpha sau khi loại biến được trình bày ở bảng 3 .

Bảng 3. Kết quả phân tích Cronbach's Alpha

\begin{tabular}{|c|c|c|c|c|}
\hline \multirow{2}{*}{$\begin{array}{c}\text { Thang } \\
\text { do }\end{array}$} & \multicolumn{2}{|c|}{ Thế hệ Y } & \multicolumn{2}{|c|}{ Thế hệ Z } \\
\hline & Cronbach's Alpha & Tương quan biến tổng & Cronbach's Alpha & Tương quan biến tổng \\
\hline TH2 & & 0,711 & & 0,779 \\
\hline TH3 & & 0,662 & & 0,801 \\
\hline TH4 & & 0,742 & & 0,807 \\
\hline TH5 & & 0,727 & & 0,750 \\
\hline TH6 & & $*$ & & 0,690 \\
\hline TT1 & & 0,680 & & 0,670 \\
\hline TT2 & 0,828 & 0,675 & 0,853 & 0,720 \\
\hline TT3 & & 0,703 & & 0,784 \\
\hline GT1 & & 0,677 & & 0,795 \\
\hline GT2 & 0,823 & 0,677 & 0,879 & 0,750 \\
\hline GT3 & & 0,679 & & 0,752 \\
\hline TL1 & & 0,711 & & 0,855 \\
\hline TL2 & 0,853 & 0,735 & 0,892 & 0,752 \\
\hline TL3 & & 0,726 & & 0,758 \\
\hline GL1 & & 0,607 & & 0,782 \\
\hline GL2 & 0,802 & 0,661 & 0,905 & 0,820 \\
\hline GL3 & & 0,677 & & 0,830 \\
\hline
\end{tabular}

(Nguồn: Kết quả xủ lý dũ liệu của tác giả)

Ghi chú: * Biến quan sát bị loại nên không xác định tương quan biến tổng

\subsection{Phân tích nhân tố khám phá EFA}

Phân tích nhân tố khám phá đối với mẫu thuộc thế hệ Y và $Z$ đều cho thấy việc phân tích nhân tố là phù hợp. Cụ thể: 
Đối với mẫu thuộc thế hệ $\mathrm{Y}: \mathrm{KMO}=0,763$; sig = 0,000; tổng phương sai trích $=73,933 \%$; Eigenvalue $=1,630$ tại 5 thành phần.

Đối với mâ̂u thuộc thế hệ $\mathrm{Z}$ : $\mathrm{KMO}=0,756$; sig $=0,000$; tổng phương sai trích $=79,117 \%$; Eigenvalue $=2,012$ tại 5 thành phần.

Hệ số tải nhân tố của các biến quan sát đều lớn hơn 0,5 và chênh lệch hệ số tải nhân tố ở các thành phần đều lớn hơn 0,3 (Bảng 4) nên thang đo đảm bảo giá trị hội tụ và giá trị phân biệt.

Bảng 4. Kết quả phân tích nhân tố khám phá EFA

\begin{tabular}{lccc}
\hline \multicolumn{1}{c}{ Thành phần } & Biến quan sát & Thế hệ Y & Thế hệ Z \\
\hline \multirow{2}{*}{ 1. Thể hiện bản thân (TH) } & TH2 & 0,797 & 0,835 \\
& TH3 & 0,719 & 0,854 \\
& TH4 & 0,812 & 0,862 \\
& TH5 & 0,803 & 0,803 \\
\hline 2. Thông tin chuyến đi & TH6 & $*$ & 0,721 \\
(TT) & TT1 & 0,772 & 0,722 \\
& TT2 & 0,773 & 0,807 \\
3. Giải trí (GTR) & TT3 & 0,816 & 0,915 \\
& GT1 & 0,802 & 0,880 \\
& GT2 & 0,768 & 0,812 \\
4. Tiết lộ (TL) & GT3 & 0,775 & 0,836 \\
& TL1 & 0,782 & 0,962 \\
5. Lưu giữ hình ảnh (GL) & TL2 & 0,861 & 0,796 \\
& TL3 & 0,799 & 0,815 \\
\hline Hệ số KMO & GL1 & 0,712 & 0,842 \\
Sig của kiểm định Bartlett & GL3 & 0,771 & 0,878 \\
Eigenvalue & & 0,803 & 0,901 \\
Tổng phương sai trích & & 0,763 & 0,756 \\
\hline
\end{tabular}

(Nguồn: Kết quả xủ lý dũ liệu của tác giả)

Chi chú: * Biến quan sát bị loại nên không xác định hệ số tải nhân tố

\subsection{Phân tích nhân tố khẳng định CFA}

Kết quả phân tích CFA (Hình 1) cho thấy các chỉ số đo lường độ phù hợp của mô hình đều đạt yêu cầu nên có thể kết luận mô hình nghiên cứu phù hợp. Cụ thể:

- Đối với thế hệ Y: Chiquare/df = 1,171; GFI = 0,956; TLI = 0,989; CFI = 0,992, RMSEA = 0,024.

- Đối với thế hệ Z: Chiquare/df = 1,554; GFI = 0,938; TLI = 0,972; CFI = 0,978, RMSEA = 0,044.

Ngoài ra, độ tin cậy tổng hợp của các nhân tố (CR) đều lớn hơn 0,6 và phương sai trích (AVE) đều lớn hơn 0,5 nên các khái niệm nghiên cứu đạt giá trị hội tụ. Căn bậc hai AVE của từng khái niệm đều lớn hơn hệ số tương quan giữa khái niệm đó với ba khái niệm còn lại (Bảng 5) nên các cấu trúc đạt giá trị phân biệt.

Bảng 5. Kết quả phân tích giá trị thang đo

\begin{tabular}{ccccccccc}
\hline & \multirow{2}{*}{ Thành phần } & \multirow{2}{*}{ CR } & \multirow{2}{*}{ AVE } & \multicolumn{5}{c}{ Tương quan giữa các thành phần } \\
\cline { 2 - 8 } & & & & TH & TT & GTR & TL & GL \\
\cline { 2 - 8 } Thế hệ Y & TH & 0,840 & 0,538 & $\mathbf{0 , 7 3 3}$ & & & & \\
& TT & 0,869 & 0,689 & 0,021 & $\mathbf{0 , 8 3 0}$ & & & \\
& GTR & 0,879 & 0,709 & 0,155 & 0,162 & $\mathbf{0 , 8 4 2}$ & & \\
& TL & 0,895 & 0,742 & 0,009 & 0,094 & 0,095 & $\mathbf{0 , 8 6 1}$ & $\mathbf{0 , 8 7 2}$ \\
\hline
\end{tabular}




\begin{tabular}{ccccccccc}
\hline & \multirow{2}{*}{ Thành phần } & \multirow{2}{*}{ CR } & \multirow{2}{*}{ AVE } & \multicolumn{5}{c}{ Tương quan giữa các thành phần } \\
\cline { 2 - 8 } & & & & TH & TT & GTR & TL & GL \\
\hline \multirow{3}{*}{ Thến hệ Z } & TH & 0,864 & 0,613 & $\mathbf{0 , 7 8 3}$ & & & & \\
& TT & 0,829 & 0,617 & 0,041 & $\mathbf{0 , 7 8 5}$ & & & \\
& GTR & 0,823 & 0,608 & 0,169 & 0,192 & $\mathbf{0 , 7 8 0}$ & & \\
& TL & 8,853 & 0,660 & 0,193 & 0,208 & 0,182 & $\mathbf{0 , 8 1 2}$ & \\
& GL & 0,805 & 0,580 & 0,318 & 0,060 & 0,210 & 0,269 & $\mathbf{0 , 7 6 2}$ \\
\hline
\end{tabular}

Ghi chú: căn bậc hai của AVE nằm trên đường chéo chính

(Nguồn: Kết quả xủ lý dũ liệu của tác giả)

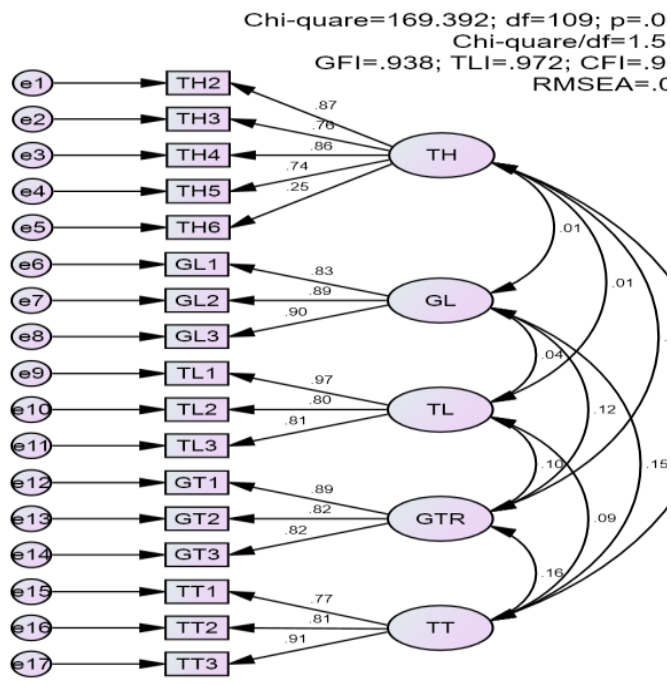

(a)

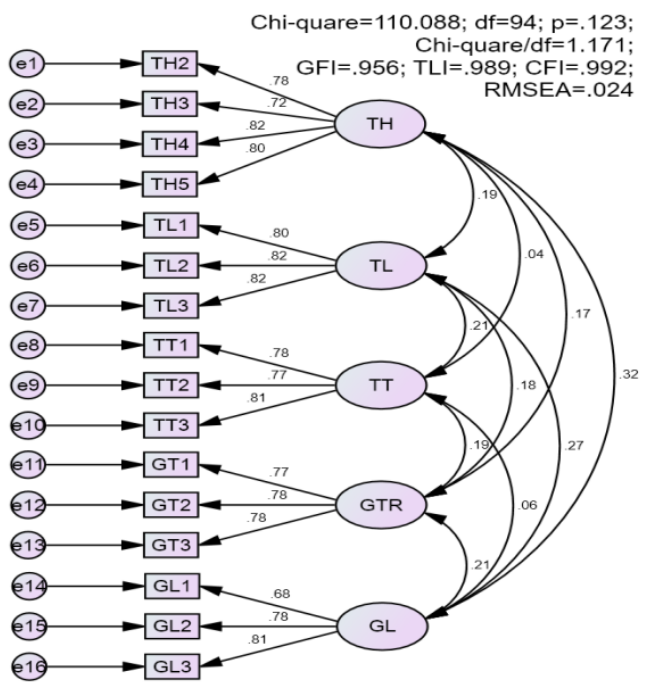

(b)

Hình 1. Kết quả phân tích CFA: (a) đối với thế hệ Y và (b) đối với thế hệ $Z$ (Nguồn: Kết quả xủ lý dũ liệu của tác giả)

\subsection{Kiểm định Independent Sample T - Tesst}

Kết quả kiểm định Independent Sample $\mathrm{T}$ - Test cho thấy có sự khác biệt giữa thế hệ Y và thế hệ $\mathrm{Z}$ về động cơ chia sẻ hình ảnh du lịch lên Facebook, cụ thể là ở 4 động cơ là động cơ thể hiện bản thân, động cơ giải trí, động cơ tiết lộ và động cơ lưu giữ hình ảnh. Trong đó, động cơ thể hiện bản thân, giải trí và tiết lộ của thế hệ $\mathrm{Z}$ luôn lớn hơn thế hệ Y. Ngược lại, động cơ lưu giữ hình ảnh của thế hệ Y lại lớn hơn thế hệ Z.

Bảng 6. Kết quả kiểm định Independent Sample T-Tesst

\begin{tabular}{lccl}
\hline \multicolumn{1}{c}{ Động co } & Sig. & Chênh lệch $(\mathbf{Z}-\mathbf{Y})$ & \multicolumn{1}{c}{ Kết luận } \\
\hline Thể hiện bản thân & 0,000 & 0,626 & Thế hệ Z lớn hơn thế hệ Y \\
Thông tin & 0,196 & 0,087 & Không có sự khác biệt \\
Giải trí & 0,000 & 0,463 & Thế hệ Z lớn hơn thế hệ Y \\
Tiết lộ & 0,000 & 0,263 & Thế hệ Z lớn hơn thế hệ Y \\
Lưu giữ hình ảnh & 0,025 & $-0,128$ & Thế hệ Y lớn hơn thế hệ Z \\
\hline
\end{tabular}

(Nguồn: Kết quả xủ lý dũ liệu của tác giả)

Đối với thế hệ Y, động cơ thông tin và động cơ lưu giữ hình ảnh là hai động cơ nổi bật, có mức đánh giá cao hơn trung bình chung, đặc biệt là động cơ lưu giữ hình ảnh (3,939 điểm). Ngược lại, động cơ tiết lộ và thể hiện bản thân thì mức độ đánh giá khá thấp, lần lượt là 3,594 điểm và 3,403 điểm.

Đối với thế hệ $Z$, động cơ giải trí là động cơ nổi bật nhất với 4,139 điểm; sau đó là động cơ thể hiện bản thân (4,029 điểm); động cơ thông tin (3,926 điểm); động cơ tiết lộ (3,856 điểm); động cơ lưu giữ hình ảnh $(3,812$ điểm). 


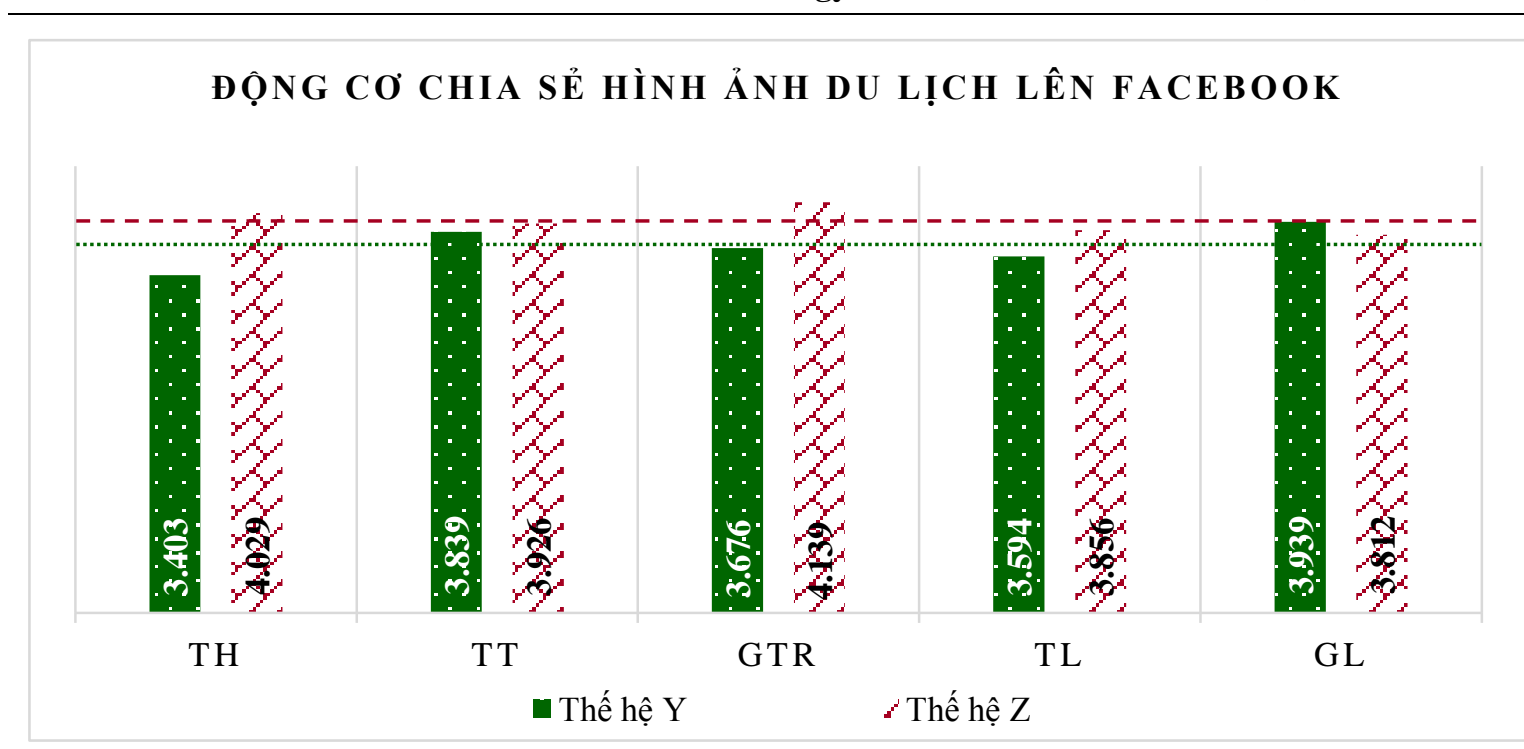

Ghi chú:

Đánh giá trung bình của thê hệ $Y$

Đánh giá trung bình của thê hệ Z

Hình 2. Động cơ chia sẻ hình ảnh du lịch lên Facebook (Nguồn: Kết quả xủ lý dũ liệu của tác giả)

\section{Kết luận và hàm ý}

Kết quả nghiên cứu cho thấy du khách ở cả 2 thế hệ là $Y$ và $Z$ đều có những động cơ chia sẻ hình ảnh du lịch lên Facebook gồm: (1) Động cơ thể hiện bản thân; (2) Động cơ thông tin; (3) Động cơ giải trí; (4) Động cơ tiết lộ; (5) Động cơ lưu giữ hình ảnh. Trong đó, động cơ thể hiện bản thân, động cơ thông tin, động cơ giải trí và động cơ tiết lộ có sự tương đồng lớn với nghiên cứu của Li (2020). Ngoài ra, nghiên cứu đã phát hiện thêm một động cơ là động cơ lưu giữ hình ảnh. Du khách chia sẻ hình ảnh du lịch lên Facebook để sau này có thể xem lại và nhớ về chuyến đi, để Facebook nhắc lại kỉ niệm về chuyến đi của họ, để dễ dàng tìm kiếm hình ảnh khi cần.

Nghiên cứu này cũng tìm ra sự khác biệt giữa thế hệ $Y$ và thế hệ $Z$ đối với động cơ chia sẻ hình ảnh du lịch lên Facebook. Hầu hết động cơ chia sẻ hình ảnh du lịch lên Facebook của thế hệ $\mathrm{Z}$ cao hơn thế hệ $\mathrm{Y}$ (ngoại trừ động cơ lưu giữ hình ảnh). Do đó, du khách thuộc thế hệ $\mathrm{Z}$ là đối tượng mà điểm đến du lịch hoặc doanh nghiệp du lịch cần đặc biệt quan tâm. Với động cơ nổi bật của thế hệ $Z$ là động cơ giải trí và động cơ thể hiện bản thân thì không gian check - in cần được thiết kế mang tính chất giải trí cao, "sang chảnh" và thể hiện được phong cách sống của giới trẻ. Du khách thuộc thế hệ Y thì động cơ nổi bật là động cơ lưu giữ hình ảnh và động cơ thông tin nên yêu cầu về không gian check - in không cao, quan trọng hơn vẫn là trải nghiệm mà du khách cảm nhận được. Điểm đến du lịch và doanh nghiệp du lịch cần chú trọng đến chất lượng phục vụ để mang lại trải nghiệm tích cực cho du khách. Từ đó, những hình ảnh mà du khách chia sẻ lên Facebook sẽ lan tỏa những điều tích cực, tạo ra hình ảnh đẹp cho điểm đến du lịch và doanh nghiệp du lịch.

Như vậy, động cơ chia sẻ hình ảnh du lịch lên Facebook của du khách rất đa dạng và có sự khác biệt giữa thế hệ Y và thế hệ Z. Để khai thác hoạt động chia sẻ hình ảnh du lịch lên Facebook nói riêng và mạng xã hội nói chung như một công cụ truyền thông, các điểm đến đến du lịch và các doanh nghiệp kinh doanh du lịch cần nhận thức được vai trò của hoạt động chụp ảnh và chia sẻ hình ảnh du lịch của du khách; thiết kế những không gian chụp ảnh phù hợp với du khách. Ngoài ra, cũng cần theo dõi và quản lý hình ảnh mà du khách đăng tải để có những chính sách, hành động kịp thời trong việc duy trì, phát triển hình ảnh điểm đến, hình ảnh doanh nghiệp. 


\section{TÀI LIẸU THAM KHẢO/ REFERENCES}

[1] B. Prideaux, L. Y. -S. Lee, and N. Tsang, "A comparison of photo-taking and online sharing behaviors of mainland Chinese and Western theme park visitors based on generation membership," Journal of Vacation Marketing, vol. 24, no. 1, pp. 29-43, 2018.

[2] F. Li, "Understanding Chinese tourists' motivations of sharing travel photos in WeChat," Tourism Management Perspectives, vol. 33, pp. 1-13, 2020.

[3] A. Malik, A. Dhir, and M. Nieminen, "Uses and gratifications of digital photo sharing on Facebook," Telematics and Informatics, vol. 33, no. 1, pp. 129-138, 2006.

[4] Advertising Vietnam, "The latest image size to post Facebook in 2021," (in Vietnamese), 2021. [Online]. Available: https://advertisingvietnam.com/kich-thuoc-anh-dang-facebook-moi-nhat-nam2021-p16757. [Accessed October 06, 2021].

[5] Omnicore, "63 Facebook Statistics You Need to Know in 2021," 2021. [Online]. Available: https://www.omnicoreagency.com/facebook-statistics/. [Accessed October 06, 2021].

[6] HR1Tech, "Update the latest figures in 2021 on social network users in Vietnam," (in Vietnamese), 2021. [Online]. Available: https://www.hr1tech.com/vi/news/cap-nhat-so-lieu-moi-nhat-2021-ve-nguoidung-mang-xa-hoi-tai-viet-nam-158.html. [Accessed October 06, 2021].

[7] P. C. Albers and W. R. James, "Travel photography," Annals of Tourism Research, vol. 15, no. 1, pp. 134-158, 1988.

[8] D. L. Groves and D. J. Timothy, "Photographic techniques and the measurement of impact and importance attributes on trip design: a case study," Loisir et Societe, vol. 24, no. 1, pp. 311-317, 2001.

[9] W. Hillman, "Travel Authenticated?: Postcards, Tourist Brochures, and Travel Photography," Tourism Analysis, vol. 12, no. 3, pp. 135-148, 2007.

[10] B. Prideaux and A. Coghlan, "Digital cameras and photo taking behaviour on the Great Barrier Reef - marketing opportunities for Reef tour operators," Journal of Vacation Marketing, vol. 16, no. 3, pp. 171-183, 2010.

[11] R. M. Chalfen, "Photography’s Roles in tourism. Some Unexplored Relationships," Annals of tourism rearch, vol. 79, pp. 435-447, 1979.

[12] B. Garrod, "Understanding the Relationship Between Tourism Destination Imagery and Tourism Photography," Journal of Travel Research, vol. 47, no. 3, pp. 346-358, 2009.

[13] S. Garlick, "Revealing the unseen: tourism, art and photography," Cultural Studies, vol. 16, no. 2, pp. 289-305, 2002.

[14] S. Pan, J. Lee, and H. Tsai, "Travel photos: Motivations, image dimensions, and affective qualities of places,” Tourism Management, vol. 40, pp. 59-69, 2014.

[15] E. Konijn, N. Sluimer, and O. Mitas, "Click to Share: Patterns in Tourist Photography and Sharing," International Journal of Tourism Research, vol. 18, no. 6, pp. 525-535, 2016.

[16] I. S. Lo, B. McKercher, A. Lo, C. Cheung, and R. Law, "Tourism and online photography," Tourism Management, vol. 32, no. 4, pp. 725-731, 2011.

[17] L. Leung, "Generational differences in content generation in social media: The roles of the gratifications sought and of narcissism," Computers in Human Behavior, vol. 29, no. 3, pp. 997-1006, 2013.

[18] A. Dhir and T. Torsheim, "Age and gender differences in photo tagging gratifications," Computers in Human Behavior, vol. 63, pp. 630-638, 2016.

[19] R. Zemke, C. Raines, and B. Filipczak, Generations at Work: Managing the Clash of Veterans, Boomers, Xers, and Nexters in Your Workplace. New York: American Management Association, 2000.

[20] Q. U. Nguyen, General Psychology Textbook. Hanoi National University of Education Publishing, 2003.

[21] Y. Cheng, J. Liang, and L. Leung, "Social network service use on mobile devices: An examination of gratifications, civic attitudes and civic engagement in China," New Media \& Society, vol. 17, no. 7, pp. 1096-1116, 2014.

[22] N. Park, K. F. Kee, and S. Valenzuela, "Being Immersed in Social Networking Environment: Facebook Groups, Uses and Gratifications, and Social Outcomes," CyberPsychology and Behavior, vol. 12, no. 6, pp. 729-733, 2009. 
[23] M. Urista, "Explaining Why Young Adults Use MySpace and Facebook Through Uses and Gratifications Theory," Human Communication, vol. 12, no. 2, pp. 215-229, 2009.

[24] E. E. Hollenbaugh and A. L. Ferris, "Facebook self-disclosure: Examining the role of traits, social cohesion, and motives," Computers in Human Behavior, vol. 30, pp. 50-58, 2014.

[25] A. Quan-Haase and A. L. Young, "Uses and gratifications of social media: A comparison of Facebook and instant messaging," Bulletin of Science Technology Society, vol. 30, no. 5, pp. 350-361, 2010.

[26] A. D. Smock, N. B. Ellison, C. Lampe, and D. Y. Wohn, "Facebook as a toolkit: A uses and gratification approach to unbundling feature use," Computers in Human Behavior, vol. 27, no. 6, pp. 2322-2329, 2011.

[27] N. K. G. Ha and N. V. Bui, Textbook of Master Degree Research Methods in Business-Updated with SmartPLS. (in Vietnamese). Finance Publishing, Hanoi, 2019.

[28] T. Hoang and N. M. N. Chu, Data Analysis with SPSS. Hong Duc Publishing, Hanoi, 2008. 